\title{
A study of the clinical characteristics and prognosis of advanced mucosal and cutaneous melanoma in a Chinese population
}

\author{
Yong Zhang ${ }^{\ddagger 1}$, Xiaomin Fü,1, Yalong Qi ${ }^{1}$ \& Quanli Gao*,1 \\ ${ }^{1}$ Department of Biotherapy, Affiliated Cancer Hospital of Zhengzhou University, 127 Dong Ming Road, Jinshui District, Zhengzhou, \\ Henan, 450008, PR China \\ *Author for correspondence: Tel.: +150 3817 1966; gaoquanli2015@126.com \\ ¥Authors contributed equally
}

\begin{abstract}
Aim: To investigate the characteristics and prognosis of melanoma in a Chinese population. Materials $\&$ methods: Total of 162 advanced melanoma patients were analyzed retrospectively. Kaplan-Meier method and Log rank test were used for survival analysis. Results: The median progression-free survival of mucosal and cutaneous melanoma patients was 13 versus 8 months $(p=0.005), 14$ versus 10 months in immunotherapy group ( $p=0.022), 6$ versus 4 months in chemotherapy group $(p=0.040)$. Age was an independent risk factor for mucosal melanoma patients. Staging and treatment regimen were independent risk factors for cutaneous melanoma patients. The lungs, liver and brain were most common metastasis locations. Conclusion: The prognosis of patients with advanced mucosal melanoma is better than cutaneous melanoma in China. There are significant differences between the two subtypes of melanoma.
\end{abstract}

First draft submitted: 7 March 2018; Accepted for publication: 5 November 2018; Published online: 4 December 2018

Keywords: chemotherapy • Chinese population • clinicopathologic characteristic • cutaneous melanoma • immunotherapy • melanoma $\bullet$ mucosal melanoma $\bullet$ prognosis

Melanoma is a highly malignant tumor. Approximately 132,000 newly diagnosed cases and about 48,000 patients died annually due to this disease worldwide [1,2]. Also, Melanoma is a disease with high heterogeneity and ethnic variations. Even in same ethnic population, there are large variations in epidemiology, clinicopathologic characteristics, genetic structures and prognosis due to different anatomicalsite, such as mucosal and cutaneous melanoma [3].

Study has shown that cutaneous melanoma is the most common subtype in Caucasians; accounting for $91.2 \%$ of melanoma cases, while mucosal melanoma accounts for only 1.3\%. Cutaneous melanoma usually occurs on the back, chest, abdomen and lower extremity skin [4]. Whereas the incidence of cutaneous melanoma is 50-70\% in Asian patients. The most common primary site is acral, for instance, sole, toes, fingers, subungual and so on. Guo et al. find that the incidence of mucosal melanoma is $22.6 \%$ in China [5]. Among which, the incidence of mucosal melanoma originating from head and neck is the highest $(55.4 \%)$, such as paranasal sinusandoral melanoma. Followed by anorectal melanoma (23.8\%), female reproductive melanoma (18.0\%) and urinary tract melanoma (2.8\%) [6]. The etiology of mucosal melanoma remains unclear. One theory is that melanoma-initiating cells originate from neural crest, undergo epithelial mesenchymal transition, metastasize to mucosal tissues and then develop into tumors [6]. Mucosal melanoma is usually seen in elderly female patients and the risk factors are still unknown [7]. Cutaneous melanoma is typically seen in male patients and the occurrence is closely associated with ultraviolet exposure [7-9]. Compared with cutaneous melanoma, patients with mucosal melanoma have higher serum lactate dehydrogenase levels (44-49\% vs 39\%) and lower PD-L1 positive rate (17-29\% vs 34\%) [10].

Most of the mucosal melanoma patients are diagnosed at a late stage because of occult site. The lymph node metastasis rate at diagnosis in mucosal melanoma is $21 \%$ at head and neck, $61 \%$ at colorectal mucosa, $23 \%$ at reproductive tract, while the lymph node metastasis rate of cutaneous melanoma is only $9 \%$ [7].

Future Medicine 
In addition, the two subtypes of melanoma show significant differences in molecular biological behavior and genetic variation. The $B R A F$ mutation occurs $40-50 \%$ in cutaneous melanoma and $10 \%$ in mucosal melanoma. However, the $c$-KIT mutation rate is much higher in mucosal melanoma than cutaneous melanoma (15-39 vs $2-10 \%$ ) [5,11-13]. Furthermore, the prognosis of mucosal melanoma is extremely poor, the median survival time is 10-13 months and 5-year overall survival (OS) rate is less than 5\% [14].

In summary, melanoma is a highly heterogeneous tumor, mucosal and cutaneous melanomas are two major subtypes with unique clinical and biological characteristics. Owing to low incidence and occult site, few studies focused on mucosal and cutaneous melanoma in China. Our aim is to perform a preliminary retrospective study on the two subtypes of melanoma; compare the differences of clinicpathologic characteristics, prognosis and metastasis mode, and also evaluate the consistency with global findings.

\section{Materials \& methods \\ Patients}

From 1 January 2010 to 31 December 2016, a total of 162 patients with advanced melanoma (stage III- IV) were collected for this study in our hospital. This study was approved by the Ethics Committee of the Affiliated Cancer Hospital of ZhengZhou University. All patients volunteered to this study and signed the consents. The patients were divided into two group: immunotherapy group and chemotherapy group.

Tumor staging was carried out according to the seventh edition of melanoma clinical staging criteria of the American Joint Committee on Cancer (AJCC), and based on imaging and pathological results. All patients were diagnosed late stage (stage III-IV) of melanoma according to AJCC. We excluded all patients who had multiple primaries.

\section{Methods}

We retrospectively analyzed the clinical data of all the 162 patients, who were classified into two groups - mucosal melanoma group and cutaneous melanoma group - based on the primary site of the tumor. Progression-free survival (PFS) was the primary end point, which was defined the length of time lived by the patient with melanoma and not get worse based on imaging results. The PFS, clinicopathologic characteristics, metastasis sites and prognosis were compared between the two groups. For subgroup analysis, patients were divided into immunotherapy group and chemotherapy group based on the treatment regimen; the PFS was also compared between the two subtypes of melanoma, even among the different primary sites of mucosal melanoma.

Patients, who accepted cytokine-induced killer (CIK) cell therapy combined with IL-2 or IFN- $\gamma$, were included in immunotherapy group. The CIK cells are heterogeneous ex vivo-expanded T lymphocytes and CD $3^{+} \mathrm{CD} 56^{+}$ $\mathrm{T}$ lymphocyte is considered as antitumor active component. The CIK cells generate from human peripheral blood mononuclear cells and incubate with IFN- $\gamma$, anti-CD3 monoclonal antibody and human IL-2. Chemotherapy group indicated that patients accepted dacarbazine $200-250 \mathrm{mg} / \mathrm{m}^{2}, \mathrm{~d} 1-3$, cisplatin $25 \mathrm{mg} / \mathrm{m}^{2}, \mathrm{~d} 1-3, \mathrm{Q} 3$; temozolomide $200 \mathrm{mg} / \mathrm{m}^{2}, \mathrm{~d} 1-5$, Q4W; or paclitaxel $175 \mathrm{mg} / \mathrm{m}^{2}, \mathrm{~d} 1$, carboplatin $200-400 \mathrm{mg} / \mathrm{m}^{2}, \mathrm{~d} 1, \mathrm{Q} 3 \mathrm{~W}$.

\section{Follow-up}

Follow-up information of patients was maintained through phone calls and from hospital archives. The cut-off follow-up date was 22 February 2017.

\section{Statistical analysis}

All analyses were performed with the Statistical Package for Social Sciences V.21.0 (SPSS Inc, IL, USA). Comparisons of the qualitative data were performed using the Pearson Chi-square $\left(\chi^{2}\right)$ test. Survival analysis was performed using the Kaplan-Meier method. Comparison between groups were performed using log-rank test. Multivariate analysis was performed using multivariate logistic regression analysis and Cox modeling, hazard ratios (HRs) was defined with $95 \%$ CIs and p-values were based on the Wald test. A p-value less than 0.05 was considered statistically significant.

\section{Results}

\section{Clinical finding}

A brief summary of clinical characteristics was shown in Table 1. The study group comprised 90 (55.6\%) female patients and $72(44.4 \%)$ male patients; 62 patients were aged $\geq 60$ years $(38.3 \%)$ and 100 patients were aged 
Table 1. Clinical characteristics of patients with mucosal melanoma and cutaneous melanoma.

\begin{tabular}{|c|c|c|c|}
\hline Characteristics & Mucosal melanoma (n/\%) & Cutaneous melanoma $(n / \%)$ & $\mathrm{p}$-value \\
\hline Total & $41(\%)$ & $121(\%)$ & \\
\hline \multicolumn{4}{|l|}{ Gender } \\
\hline Male & $15(36.6)$ & $57(47.1)$ & 0.241 \\
\hline Female & $26(63.4)$ & $64(52.9)$ & \\
\hline \multicolumn{4}{|l|}{ Age (years) } \\
\hline$<60$ & $21(51.2)$ & 79 (65.3) & 0.109 \\
\hline \multicolumn{4}{|l|}{ Smoking history } \\
\hline Yes & $11(26.8)$ & $40(33.1)$ & 0.458 \\
\hline No & $30(73.2)$ & $81(66.9)$ & \\
\hline \multicolumn{4}{|l|}{ Drinking history } \\
\hline Yes & $9(22.0)$ & $32(26.4)$ & 0.567 \\
\hline \multicolumn{4}{|l|}{ Metastasis site } \\
\hline Brain & $4(9.8)$ & $10(8.3)$ & 0.991 \\
\hline Lung & $12(29.3)$ & $35(28.9)$ & \\
\hline Liver & $6(14.6)$ & $19(15.7)$ & \\
\hline Others & $19(46.3)$ & $57(47.1)$ & \\
\hline \multicolumn{4}{|c|}{ Treatment regimen } \\
\hline Immunotherapy & $31(75.6)$ & 87 (71.9) & 0.644 \\
\hline Chemotherapy & $10(24.4)$ & $34(28.1)$ & \\
\hline
\end{tabular}

$<60$ years (61.7\%). Mucosal melanoma was noted in 41 patients $(25.3 \%)$ with the following anatomical distribution: total of 22 patients at head and neck region (nasal cavity: 15 cases, oral cavity: 7 cases); 11 patients at rectum; seven patients at vagina and one patient at urinary tract. There were 121 cases $(74.7 \%)$ of cutaneous melanoma including skin of the limbs and trunk. There were 53 patients $(32.7 \%)$ with stage III melanoma and 109 patients with stage IV melanoma (67.3\%). A total of 118 patients $(72.8 \%)$ were treated with immunotherapy, while 44 patients $(27.2 \%)$ were treated with chemotherapy alone. Total of 118 patients in CIK group received 1-35 infusions, with a mean and median course of 2.0 and 1.5, respectively. The median number of CIK cells for each dose was $4.7 \times 10^{9}$ (range from $3.5 \times 10^{9}$ to $5.0 \times 10^{9}$ ).

\section{Comparison of the clinical characteristics between patients with mucosal melanoma \& cutaneous melanoma}

There were no significant differences in age, gender, smoking, alcohol intake, clinical stage, location of metastases and treatment regimens between patients with mucosal melanoma and cutaneous melanoma $(\mathrm{p}>0.05)($ Table 1$)$.

\section{Comparison of the survival between patients with mucosal melanoma \& cutaneous melanoma}

The median PFS of all the patients was 9 months, the median PFS of patients with mucosal and cutaneous melanoma was 13 months and 8 months, respectively $(\mathrm{p}=0.005$, Figure 1$)$. Further subgroup analysis found that, in patients treated with immunotherapy, the median PFS of patients with mucosal and cutaneous melanoma was 14 months and 10 months, respectively $(p=0.022$, Figure 2$)$. In patients treated with chemotherapy, the median PFS was 6 months compared with and 4 months in patients with mucosal and cutaneous melanoma $(p=0.040$, Figure 3).

Univariate \& multivariate survival analyses of factors affecting the prognosis of patients Univariate analysis showed correlation between age and the prognosis of patients with late-stage mucosal melanoma $(\mathrm{p}<0.05$, Table 2$)$, while gender, clinical stage and treatment regimen were associated with the prognosis of patients 

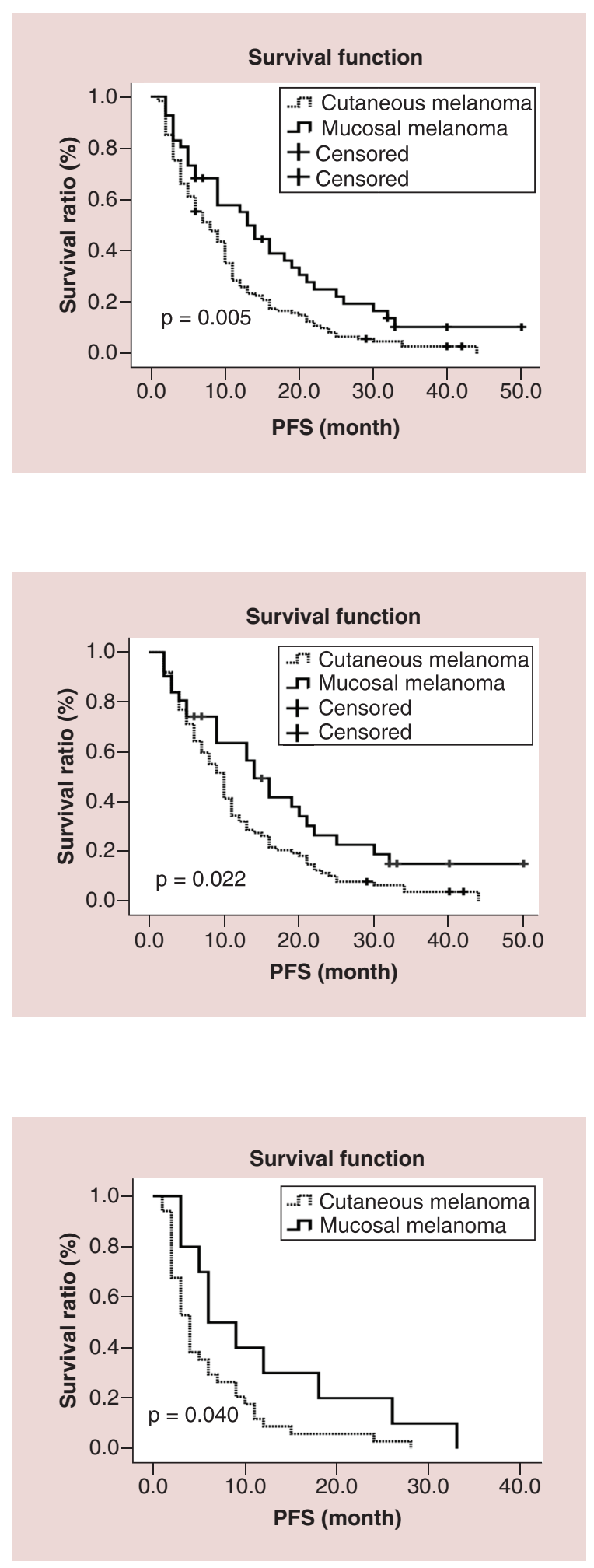

Figure 1. The curves of progression free survival for patients with mucosal and cutaneous melanoma. Kaplan-Meier curves of PFS for all patients. The overall median PFS of all patients was 9 months, and the median PFS of patients with mucosal and cutaneous melanomas was 13 months and 8 months, respectively $(p=0.005)$. PFS: Progression-free survival.

Figure 2. The curves of progression free survival for patients treated with immunotherapy. Kaplan-Meier curves of PFS for patients treated with immunotherapy. The median PFS of patients with mucosal and cutaneous melanomas was 14 months and 10 months, respectively $(\mathrm{p}=0.022)$.

PFS: Progression-free survival.

Figure 3. The curves of progression free survival for patients treated with chemotherapy. Kaplan-Meier curves of PFS for patients treated with chemotherapy. The median PFS of patients with mucosal and cutaneous melanomas was 6 months and 4 months, respectively $(p=0.040)$.

PFS: Progression-free survival.

with cutaneous melanoma $(\mathrm{p}<0.05$, Table 2$)$. Multivariate analysis showed that age was an independent factor affecting the prognosis of patients with late-stage mucosal melanoma ( $p<0.05$, Table 3$)$, and clinical stage and treatment regimen were found to be independent factors associated with the prognosis of patients with cutaneous melanoma (both $\mathrm{p}<0.05$, Table 3). 
Table 2. Univariate analysis of clinical factors associated with median progression free survival in patients with

\section{melanoma.}

Characteristics

Mucosal melanoma

Cases

Total

41

Gender

Male

Female

Age (years)

$<60$

$\geq 60$

Smoking history

Yes

No

Drinking history

Yes

No

Stage

III

IV

Anatomical site

Head and neck

Rectum

Vagina

Others

Treatment regimen

Immunotherapy

Chemotherapy

Head and neck: nasal cavity and oral cavity.

mPFS: Median progression-free survival.

19

26

14

14

6

mPFS $\quad$ p-value \begin{tabular}{ccc}
\multicolumn{2}{c}{ Cutaneous melanoma } \\
\end{tabular}

$13 \quad 121 \quad 8$

$\begin{array}{llll}16 & 0.953 & 57 & 6 \\ 9 & & 64 & 8\end{array}$

0.007

$79 \quad 7$

8

0.960

$40 \quad 8$

0.311

$12 \quad 81$

0.701

0.432

$\begin{array}{lll}25 & 32 & 6 \\ 13 & 89 & 6\end{array}$

0.823

40

6

0.513

$13 \quad 81$

0.000

$13 \quad 0.513$

0.206

87

10

0.000

$6 \quad 34$

4

$7 \quad 0.432$

$\begin{array}{ll}6 & \\ 12 & 0.000 \\ 6 & \end{array}$

4

Table 3. Multivariate Cox-regression analysis of clinical factors associated with median progression free survival in patients with melanoma.

\begin{tabular}{|c|c|c|c|c|}
\hline \multirow[t]{2}{*}{ Characteristics } & \multicolumn{2}{|c|}{ Mucosal melanoma } & \multicolumn{2}{|c|}{ Cutaneous melanoma } \\
\hline & $R R(95 \% C l)$ & p-value & $R R(95 \% C l)$ & p-value \\
\hline Age (years) & $2.509(1.242-5.062)$ & 0.010 & & \\
\hline Stage & & & $2.577(1.691-3.928)$ & 0.000 \\
\hline Treatment regimen & & & $1.935(1.276-2.934)$ & 0.002 \\
\hline
\end{tabular}

Comparison of common metastasis sites between mucosal \& cutaneous melanoma

The most common metastasis site in patients with mucosal and cutaneous melanoma was lung (29.3 vs $28.9 \%)$, followed by liver (14.6 vs $12.7 \%$ ) and brain ( 9.8 vs $8.3 \%$ ), there was no significant difference between two subtypes of melanoma $(\mathrm{p}=0.991)$.

\section{Discussion}

In this study, we compared the differences of prognosis between patients with late-stage mucosal and cutaneous melanoma. Concurrently, we examined the clinicpathological characteristics about both subtypes of melanoma and factors affecting the prognosis of the patients. We found that in Chinese patients with mucosal and cutaneous melanoma, there were great differences in prognosis and affecting factors compared with Western populations. It verifies the truth that melanoma is a malignant tumor with high heterogeneity. 
In our study, differences were observed between the prognosis of mucosal and cutaneous melanoma. The PFS of patients with mucosal melanoma was significantly longer than those with cutaneous melanoma $(p=0.005)$. In order to exclude the effects of treatment regimen, we further analyzed the PFS of patients in immunotherapy group and chemotherapy group. We found that, regardless of immunotherapy or chemotherapy, the PFS of patients with mucosal melanoma was superior to those with cutaneous melanoma. Therefore, it can be inferred that mucosal melanoma may have better prognosis thancutaneous melanoma in China.

Researchers in different countries have carried out studies on the prognosis of patients with different subtypes of melanoma. In China, Guo et al. retrospectively analyzed 552 patients with malignant melanoma; found no significant differences in disease-free survival and OS between different subtypes of patients with mucosal melanoma, acral lentiginous melanoma, head and neck melanoma [15]. A retrospective analysis in South Korea: all 95 patients were treated with dacarbazine chemotherapy, among which 28 (29.5\%) cases were mucosal melanoma, 23 (24.2\%) cases were cutaneous melanoma and $37(38.9 \%)$ cases were acral lentiginous melanoma. Results showed that the objective response rate (ORR) of mucosal and cutaneous melanoma were 20 and $30 \%$, respectively $(p=0.206)$ and there was no significant difference in OS between the two groups [16]. Another clinical study of South Korea, involving 32 patients who experienced treatment failure from dacarbazine, found that there was no significant difference in PFS between mucosal and cutaneous melanoma [17]. These studies suggest that the prognosis of Asian patients with mucosal melanoma is similar to those with cutaneous melanoma. However, these studies did not analyze patients with late-stage melanoma (stage III and IV). In our study, we examined patients with late-stage melanoma, excluded the possible effects of treatment regimen, and come to the conclusion that the PFS of patients with late-stage mucosal malignant melanoma is significantly longer than the patients with cutaneous malignant melanoma.

Most of the non-Asian studies suggest that compared with cutaneous melanoma, mucosal melanoma is a subtype with higher degree of malignancy, poorer prognosis, shorter PFS and OS, worse response to both conventional treatments (surgery, chemotherapy) and newly targeted therapies such as ipilimumab [7,10,18,19]. In addition, for mucosal malignant melanoma patients, the median OS time is only 10-13 months and the 5-year OS rate is less than 5\% [14]. Recently, Larkin et al. have reported a Phase III clinical trial on melanoma conducted in the UK [10]. The study enrolled 1296 patients, of which 121 (9.3\%) were patients with mucosal melanoma. The patients were divided into three groups: nivolumab alone, ipilimumab alone and nivolumab combined with ipilimumab. In the nivolumab group, the median PFS of patients with mucosal and cutaneous melanoma was 3.0 and 6.2 months, respectively, and the ORR was 23.3 and $40.9 \%$, respectively. In the ipilimumab group, the median PFS of patients with mucosal and cutaneous melanoma was 2.7 and 3.9 months, respectively, and the ORR was 8.3 and $21.2 \%$, respectively. In the nivolumab + ipilimumab group, the median PFS of patients with mucosal and cutaneous melanoma was 5.9 and 11.7 months, respectively, and the ORR was 37.1 and $60.4 \%$, respectively. Thus, regardless of the treatment regimen, large differences in PFS and ORR exist between patients with mucosal melanoma and those with cutaneous melanoma; patients with mucosal melanoma have poorer prognosis. Reasons of the differences in treatment effects between the two subtypes of melanoma are still not apparent, and the distincts between different populations need to be further explored. This may be due to biases originating sample size, variations in biological responses such as $B R A F$ and $c$-Kit mutations, and different clinicpathological characteristics between the two subtypes. Data from large-scale prospective studies could explain the underlying mechanisms of these differences.

In this study, we analyzed many factors affecting the prognosis of patients and found large differences between the subtypes. For mucosal melanoma patients, multivariate analysis indicated that age was the only independent risk factor affecting the PFS of patients, while gender, clinical stage, primary site and treatment regimen did not have any effects on PFS. For cutaneous melanoma, multivariate analysis found that clinical stage and treatment regimen were independent factors affecting the PFS of patients.

Age is an important epidemiological data for disease; however, in many cancers such as liver cancer [20], colorectal cancer [21] and small-cell lung cancer [22], it has no significant effects on prognosis. Interestingly, our study found that age was an independent factor which affects the prognosis of late-stage mucosal melanoma. For patients with late-stage mucosal melanoma, the PFS of patients less than 60 years old was significantly better than those more than 60 (18 vs 6 months, $\mathrm{p}=0.007$ ). Schaefer et al. [11] analyzed the prognosis of 75 patients with mucosal melanoma and found that the median OS of patients aged $<60$ years was 52 months, and the patients aged $\geq 60$ years had a median OS of 24 months. Kim et al. [23,24] verified that age was an independent risk factor for mucosal melanoma. 
A surprising result from our study is that there were no significant differences in OS among mucosal melanoma originating from the head and neck, rectum, vagina and other sites. Recently, Chinese researchers Guo et al. published a new study of 706 patients with mucosal melanoma, of which $26.5 \%$ had digestive tract melanoma, $23 \%$ had melanoma in nose and paranasal sinuses, $22.5 \%$ had gynecological melanoma (cervix and vagina), $15 \%$ had oral melanoma and 5\% had melanoma of the urinary tract. No significant differences in 1-, 2- and 5-year survival data were found in this study [25]. An international study reported that the 5-year survival rate of patients with head and neck melanoma was the highest compared with colorectal and female genital tract melanoma.The 5-year survival rate was 31.7, 19.8 and $11.4 \%$, respectively [8]. These results suggest that a large variation may exist between Chinese and Western patients with mucosal melanoma.

Another unexpected finding was that clinical stage had no significant effects on PFS of late-stage mucosal melanoma patients. Staging is important for pathological characterization of malignant tumors including melanoma, and late clinical stage generally suggests a higher degree of malignancy and poorer prognosis [11,26-28]. The following reasons may explain the differences between the present study and other international investigations. First, previous studies are mostly from Western countries and the constitution of disease differs greatly from those in China, as they have a larger number of patients with nonmucosal melanoma. Second, there are not unified staging methods for mucosal melanoma until now. Many studies, including ours, have used the AJCC staging method. The AJCC staging method is predominantly used for cutaneous melanoma. Increasing number of studies have reported the deficiencies of AJCC staging for staging of mucosal melanoma and indicate that clinical stage may not be a good indication for the survival of patients $[6,8,11,13]$, including two retrospective studies from China $[15,29]$. Some researchers have proposed classifying mucosal malignant melanoma into stages I, II and III using the Ballantyne's staging system (stage I: localized disease; stage II: local metastasis; stage III: distal metastasis). This method may provide more reliable prognostic information [30,31]. Our findings suggest that it is necessary to update the staging of highly heterogenous tumors including melanoma, especially in the Asian population and at different anatomical sites, which may be of better significance in guiding the treatment and judging the prognosis of the patients.

We carried out a preliminary analysis of the metastatic sites for mucosal and cutaneous melanoma patients, and our results showed that the most common metastatic site was lung $(29.3$ vs $28.9 \%)$, followed by liver (14.6 vs $12.7 \%$ ) and brain $(9.8$ vs $8.3 \%$ ). Therefore, during therapy, the focus should not only be on the treatment of primary disease, but also on regular examinations of lung, liver and brain metastasis for early detection and therapeutic interventions.

As many patients missed pathologic details at local hospital and diagnosed with late stage when they came to our hospital, this study did not discuss the relationship between tumor thickness, ulceration, growth pattern and prognosis. Also, our study has limitations such as treatment selection bias due to retrospective study and small sample size, particularly patients with mucosal melanoma. In addition, owing to loss of follow-up information, we did not get OS data of some patients. Studies showed that the presence of melanin can affect the behavior of melanoma cells and prognosis [32,33], but we did not pay attention to it due to the limitation of pathological items. These limitations may have some influence on our results.

\section{Conclusion}

In summary, melanoma is a type of malignant tumor with high heterogeneity. There are great differences in characteristics of melanoma between China and other countries, also big variances exist between the two subtypes of the disease. Therefore, we should endeavor to explore epidemiological information, pathological characteristics and prognostic indicators of every subtype, and study the molecular or genetic modification to shed light on the possible reasons for these differences. This will aid in establishing individualized clinical strategies for different patients.

\section{Financial \& competing interests disclosure}

This work was supported by the National Key Technology R\&D Program (grant number 2015BAI12B12) and National Clinical Research Center for Cancer, Henan Provincial Programs for Medical Science and Technology Development (grant number 201702239), Henan Medical Science and Technique Foundation (grant number 201701030, 201702239) and Henan Provincial Scientific and Technological Project (grant number 162300410095). The authors have no other relevant affiliations or financial involvement with any organization or entity with a financial interest in or financial conflict with with subject matter or materials discussed in the manuscript apart from those disclosed.

No writing assistance was utilized in the production of this manuscript. 


\section{Ethical conduct of research}

The authors state that they have obtained appropriate institutional review board approval or have followed the principles outlined in the Declaration of Helsinki for all human or animal experimental investigations. In addition, for investigations involving human subjects, informed consent has been obtained from the participants involved.

\section{Summary points}

- Melanoma is a malignant tumor with high heterogeneity.

- The prognosis of patients with advanced mucosal melanoma is better than cutaneous melanoma in China.

- The most common metastasis sites were lung, liver and brain for both subtypes of melanoma.

- Age was an independent risk factor for patients with mucosal melanoma.

- Staging and treatment regimen were independent risk factors for patients with cutaneous melanoma.

- There are great differences between Chinese and Eastern patients, as well as the two subtypes of melanoma.

\section{References}

1. American Cancer Society. Cancer facts and figures 2014 www_x000d_.cancer.org/acs/groups/content/@research/documents/webcontent/acspc-042151.pdf

2. World Health Organization. Ultraviolet radiation and the INTERSUN Program: skin cancers. www.who.int/uv/faq/skincancer/en/index1.html

3. Cormier JN, Xing Y, Ding M et al. Ethnic differences among patients with cutaneous melanoma. Arch. Intern. Med. 166(17), 1907-1914 (2006).

4. Sahoo MR, Gowda MS, Kaladagi RM. Primary amelanotic melanoma of the rectum mimicking adenocarcinoma. Am. J. Case. Rep. 14, 280-283 (2013).

5. Wang X, Si L, Guo J. Treatment algorithm of metastatic mucosal melanoma. Chin. Clin. Oncol. 3(3), 38 (2014).

6. Tacastacas JD, Bray J, Cohen YK et al. Update on primary mucosal melanoma. J. Am. Acad. Dermatol. 71(2), 366-375 (2014).

7. Carvajal RD, Spencer SA, Lydiatt W. Mucosal melanoma: a clinically and biologically unique disease entity. J. Natl. Compr. Canc. Netw. 10(3), 345-356 (2012).

8. Mihajlovic M, Vlajkvic S, Jovanovic P et al. Primary mucosal melanomas:a comprehensive review. Int. J. Clin. Exp. Pathol. 5(8), 739-753 (2015).

9. Postow MA, Hamid O, Carvajal RD. Mucosal melanoma: pathogenesis, clinical behavior, and management. Curr. Oncol. Rep. 14(5), 441-448 (2012).

10. D'Angelo SP, Larkin J, Sosman JA et al. Efficacy and safety of nivolumab alone or in combination with ipilimumab in patients with mucosal melanoma: a pooled analysis. J. Clin. Oncol. 35(2), 226-235 (2017).

11. Schaefer T, Satzger I, Gutzmer R. Clinics, prognosis and new therapeutic options in patients with mucosal melanoma: a retrospective analysis of 75 patients. Medicine (Baltimore) 96(1), e5753 (2017).

12. Si L, Wang X, Guo J. Genotyping of mucosal melanoma. Chin. Clin. Oncol. 3(3), 34 (2014).

13. Shoushtari AN, Bluth MJ, Goldman DA et al. Clinical features and response to systemic therapy in a historical cohort of advanced or unresectable mucosal melanoma. Melanoma Res. 27(1), 57-64 (2017).

14. Li B, Lei W, Shao K et al. Characteristics and prognosis of primary malignant melanoma of the esophagus. Melanoma Res. 17(4), 239-242 (2017).

15. Chi Z, Li S, Sheng X et al. Clinical presentation, histology, and prognoses of malignant melanoma in ethnic Chinese: a study of 522 consecutive cases. BMC Cancer 11, 85 (2011).

16. Yi JH, Yi SY, Lee HR et al. Dacarbazine-based chemotherapy as first-line treatment in noncutaneous metastatic melanoma: multicenter, retrospective analysis in Asia. Melanoma Res. 21(3), 223-227 (2011).

17. Chang W, Lee SJ, Park S et al. Effect of paclitaxel/carboplatin salvage chemotherapy in noncutaneous versus cutaneous metastatic melanoma. Melanoma Res. 23(2), 147-151 (2013).

18. Bitas C, Shoushtari AN, Bluth MJ et al. The Memorial Sloan Kettering Cancer Center (MSKCC)_x000d_experience of systemic therapy in mucosal melanoma. J. Clin. Oncol. 32)15), (abstr 9073) (2014).

19. Postow MA, Luke JJ, Bluth MJ et al. Ipilimumab for patients with advanced mucosal melanoma. Oncologist 18(6), 726-732 (2013).

20. Hu B, Yang XR, Xu Y et al. Systemic immune-inflammation index predicts prognosis of patients after curative resection for hepatocellular carcinoma. Clin. Cancer Res. 20(23), 6212-6222 (2014).

21. Li H, Song J, Cao M et al. Preoperative neutrophil-to-lymphocyte ratio is a more valuable prognostic factor than platelet-to-lymphocyte ratio for nonmetastatic rectal cancer. Int. Immunopharmacol. 40, 327-331 (2016). 
22. Hong X, Cui B, Wang M et al. Systemic immune-inflammation index, based on platelet counts and neutrophil-lymphocyte ratio, is useful for predicting prognosis in small cell lung cancer. Tohoku J. Exp. Med. 236(4), 297-304 (2015).

23. Kim HS, Kim EK, Jun HJ et al. Noncutaneous malignant melanoma: a prognostic model from a retrospective multicenter study. $B M C$ Cancer 10, 167 (2010).

24. Jethanamest D, Vila PM, Sikora AG et al. Predictors of survival in mucosal melanoma of the head and neck. Ann. Surg. Oncol. 18(10), 2748-2756 (2011).

25. Lian B, Cui CL, Guo J et al. The natural history and patterns of metastases from mucosal melanoma: an analysis of 706 prospectively-followed patients. Ann. Oncol. 28(4), 868-873 (2017).

26. Keller DS, Thomay AA, Gaughan J et al. Outcomes in patients with mucosal melanomas. J. Surg. Oncol. 108(8), 516-520 (2013).

27. Chen H, Cai Y, Liu Y et al. Incidence, surgical treatment, and prognosis of anorectal melanoma from 1973 to 2011: a population-based SEER analysis. Medicine (Baltimore) 95(7), e2770 (2016).

28. Weiss SA, Hanniford D, Hernando E et al. Revisiting determinants of prognosis in cutanous melanoma. Cancer 121(23), 4108-4123 (2015).

29. Zhu W, Zou B, Wang S. Clinicopathological features and prognosis of sinonasal mucosal malignant melanoma: a retrospective study of 83 cases in a Chinese population. ORL. J. Otorhinolaryngol. Relat. Spec. 78(2), 94-104 (2016).

30. Koivunen P, Back L, Pukkila M et al. Accuracy of the current TNM classification in predicting survival in patients with sinonasal mucosal melanoma. Laryngoscope 122(8), 1734-1738 (2012).

31. Lopez F, Rodrigo JP, Cardesa A et al. Update on primary head and neck mucosal melanoma. Head Neck 38(1), 147-155 (2016).

32. Brozyna AA, Jóźwicki W, Roszkowski K et al. Melanin content in melanoma metastases affects the outcome of radiotherapy. Oncotarget 7(14), 17844-17853 (2016).

33. Slominski RM, Zmijewski MA, Slominski AT. The role of melanin pigment in melanoma. Exp. Dermatol. 24(4), 258-259 (2015). 